\title{
34
}

\section{QoS support for distributed multimedia communications}

\author{
F. García ${ }^{\circ}$ D. Hutchison*, A. Mauthe* and N. Yeadon* \\ *Computing Department, Lancaster University \\ Lancaster LA1 4YR, U. K., \\ Tel: (++44) 1524/65201, Fax: (++44) 1524/ 593608, \\ email: (andreas,njy,dh)@comp.lancs.ac.uk \\ ${ }^{\circ}$ Hewlett-Packard Laboratories, \\ Telecoms Management Department \\ Filton Road, Stoke Gifford \\ Bristol BS12 6QZ U. K. \\ Tel: (++44) 117/922 8817 \\ e-mail:fg@hplb.hpl.hp.com
}

\begin{abstract}
The nature of distributed multimedia applications is such that they require multipeer communication support mechanisms. Multimedia traffic needs to be delivered to end-systems, networks and end-users in a form that they can handle while satisfying the constraints imposed by the multimedia application. Quality of Service (QoS) mechanisms are required that can ensure full quality media playout at high-performance workstations while at the same time providing appropriately filtered lower quality media for playout at low-end systems. Existing multicast support mechanisms are deficient for this purpose, in a heterogeneous environment, because they work on a lowest common denominator premise where the quality provided depends on the least capable link or node involved in the multicast session. In this paper we discuss a QoS model to provide receiver-dependent QoS based on filtering techniques, and we present initial experimental results.
\end{abstract}

\section{Keywords}

Distributed multimedia, QoS, group communication, filtering

\section{INTRODUCTION}

Computing platforms are more and more frequently used to support man-machine and interpersonal communication. The wish to communicate as naturally as possible has provoked two trends in telecommunications research: first, the integration of continuous media 
characterised by very large bandwidth requirements and timing constraints; and second the move from simple peer-to-peer communications to group or multipeer communications. The former requires resource reservation and QoS management to ensure that time constraints of audio and video are met. The latter requires the delivery of identical data units to multiple recipients. Plenty of research has tackled both trends independently, but QoS support for multipeer continuous media data communications is only just beginning to receive the attention it deserves (Pasquale, 1992), (Henckel, 1994), (Mathy, 1994).

Deriving satisfactory end-to-end QoS support within multicast groups for continuous media data services is a challenging research issue. While a common quality agreement for data capture, transfer and display between peer users and provider can potentially be negotiated and maintained in peer-to-peer communications this task becomes overwhelming when considering peer-to-multipeer communications. The major problem lies in resolving heterogeneity issues. These issues, in an open wide area environment, are evident in both end-systems and networks. Another factor which influences these QoS support mechanisms is that distinct users have different requirements. The perception of good video or audio quality is, ultimately, user dependent. We also need to determine what constitutes successful data transmission for multicast continuous media data. These problems are all related, especially when QoS has to be maintained.

The emerging ISO Open Distributed Processing (ODP) framework is addressing similar problems. Its main concern is to provide standards for distributed processing in an open environment, across end systems, but also within end systems. Although multimedia is now being considered, the current ODP standards were not originally intended to deal with continuous media. The classic ODP invocation abstraction for instances is unsuitable since it does not have a concept for information flowing over time. Also, the original ODP engineering model was not designed to provide the required sustained system support over a period of time to maintain the isochronous nature of continuous media. In general, the current ODP model lacks sufficient support of QoS, synchronisation and groups (Coulson, 1995). Proposals to enhance ODP to consider QoS are being discussed (Stefani, 1993).

Previous work at Lancaster, in the area of QoS support for distributed multimedia applications, has concentrated on resource management strategies for an extended Chorus micro-kernel (Robin, 1994) and a Quality of Service Architecture (QoS-A) (Campbell, 1994) which proposes a framework to specify and implement the required performance properties of multimedia applications over ATM networks. This work is however still concentrated on supporting peer-to-peer communications. Presently, we are motivated by the Lancaster environment which consists of PCs, workstations and specialised multimedia enhanced devices connected by ATM, Ethernet, mobile, and proprietary high-speed networks.

In this paper we present a model for the establishment and management of continuous media data flows between a single sender (source) and multiple receivers (clients). The QoS requirements of individual receivers are met through the utilisation of filtering techniques. Our filters operate on encoded data streams and can adapt a data flow to meet the special needs of single users. To determine the success of the data transmission we introduce the concept of multipeer integrity. According to this concept data delivery is deemed successful if certain integrity conditions are met.

This paper is organised in six sections. In section two we discuss the characteristics and requirements of multipeer data flows. Section three introduces our flow management model. The fourth section outlines the current protocol suite. Subsequently, in section five, we present initial experimental results obtained from the use of or currently implemented filter operations. Finally in section six we provide conclusions.

\section{MULTIPEER DATA FLOWS}

Entities (e.g. set of users, object entities, set of processors, etc.) geographically distributed across an open communications environment can communicate with one another in multiple 
ways. The simplest communication form is the one-to-one binding (point-to-point or unicast), where one entity transmits and the other receives. An alternative form of communication is the one-to-many binding (point-to-multipoint or multicast). Here, one entity transmits and multiple entities receive. These two simple communication paradigms, point-to-point and point-tomultipoint, form the basic building blocks through which complex communication topologies (e.g. many-to-many) between multiple entities can be established. Such communication topologies are commonly referred to as multipeer communications.

A continuous media data flow is a connection between a single sender and multiple receivers established for the purpose of transmitting continuous media data, i.e. audio and video. Flow characterisation is used to represent and convey QoS requirements to the various protocols involved in peer-to-peer and peer-to-multipeer communications. Requirements are expressed through the aid of quantitative parameters and qualitative attributes. These are encapsulated into a flow spec (Partridge, 1993) and may then be distributed to peer-users, network and endsystem resource management protocols to facilitate peer-to-peer and peer-to-multipeer context establishment and maintenance. In our model we work with pre-defined specifications of video/ audio flows ideally suited to specific application requirements and set of devices. These predefined specifications, uniquely identified and version controlled, are maintained in a flow library.

\subsection{Multipeer communications}

Multicast functionality and support mechanisms have been proposed in the past. At present these mechanisms are mainly employed to support fault tolerance and task or data distribution. They inherently deal with file transfer and applications which require delivery of a single self contained message or task to one or more recipients. On the other hand, QoS support mechanisms for continuous media usually deals with point-to-point communications only, point-to-multipoint QoS support mechanisms are only just beginning to be considered.

Digital audio and video services are characterised by a continual delivery of segments over a finite period of time from the commencement of the audio or video sequence. Each segment of this sequence is strongly time related to the previous segment. To preserve continuity in playout at a receiver, successive segments have to be delivered within tight timing constraints otherwise the service becomes disrupted. When developing point-to-multipoint QoS support mechanisms, time integrity, resource utilisation, reliability and what we call multipoint integrity need special consideration.

Time integrity is crucial for continuous media, it has to be preserved in media transfer and delivery to multiple recipients. Of interest are mechanisms which support throughput and delay (including delay jitter). Throughput and delay requirements are typically recipient dependent. Each recipient can have distinct processing capabilities and available buffer resources to deal with these requirements. However, some applications may need to tightly control the playout of media at the disparate receivers ensuring that this playout is synchronised. This implies that delays need to be equally maintained across all recipients. Throughput may be reduced while ensuring that the start and end of the respective media objects at each receiver is synchronised.

Considering the sheer volumes of data likely to be transmitted, QoS support mechanisms have to be highly efficient to ensure an optimal resource utilisation in terms of processing power (including scheduling), memory, and bandwidth. For example, the use of proper network multicast facilities (if available) so that multiple connections to the individual recipients are not required. This would minimise data copying at the transmitting end-system and would reduce bandwidth requirements. If multicasting facilities are not supported by the underlying network, the level of data copying and bandwidth requirements will be proportional to the number of recipients.

Reliability deals with the type of error control schemes employed to handle experienced error rate. Multipoint integrity specifies conditions on the number and/ or identity of recipients who are required to consider the data transmission successful. In general, any developed QoS 
support mechanisms need to be flexible, allowing geographically distinct recipients to tailor these mechanisms in accordance to their individual needs or capabilities.

\subsection{Flow characterisation}

The flow spec employed is an extension of the previous Lancaster flow spec described in (Campbell, 1994). The extensions allow for the identification of encoding types, filters, multipeer integrity and sub-flow identification. Some of the more important characteristics identifiable in the revised flow spec are highlighted here.

The quantitative QoS parameters considered in the characterisation of a continuous media flow include throughput, transit delay, transit delay jitter and error rates. Each of these parameters, in turn, may be represented by a range of values expressing maximum, average and minimum requirements. Each maximum, average and minimum value may also be bounded for QoS negotiation purposes (SC21/1, 1995), (Danthine, 1992).

While this may lead to a complete specification of quantitative QoS requirements it is felt that the QoS mechanisms required to perform the negotiation between peer-users and providers for continuous media services in a heterogeneous environment will be overly complex. Also, it is not feasible to assume that such complete specifications can be obtained for a particular continuous media flow. A minimal quantitative QoS parameter set is therefore employed for characterising continuous media flows. Don't care or don't know values may be attached to QoS values where appropriate.

Agreement levels relate to the type of service support required from the underlying provider in meeting the quantitative QoS requirements expressed for throughput, transit delay, transit delay jitter and error rates. Three levels of agreement are defined which a user may request including best effort, compulsory and guaranteed (SC21/1, 1995). Any user chosen level has to be agreed by the provider at the QoS establishment phase. Thus, the provider need not agree with the requested level. The nature of agreement reached will depend on available computing platform resources and underlying network support services.

Threshold QoS values are special case parameters. If a user identifies a threshold value, the provider is required to monitor and perform an associated action whenever the threshold is reached. Making this value a special case allows a service provider to implement adaptive QoS mechanisms which may be employed to support best effort or compulsory services.

A reliability clause is used to allow a user to specify the type of error control mechanism to be instantiated by the provider on the detection of transmission errors including lost, corrupted or duplicated PDUs. Applications will express reliability requirements depending on their sensitivity to such errors in transmission. The choice of a reliability mechanism depends on the importance of the media and the characteristics of the encoding algorithm. For some encoding algorithms, if nothing is done about a transmission error, the error may propagate over several media units or the decoder's clock may lose synchronisation.

Also, distinct reliability mechanisms may be selected independently for sub-flows of a hierarchical encoding. For example, the highest priority sub-flow from which the minimum quality media signal can be reproduced can have both error recovery and announcement mechanisms instantiated. For the lower priority sub-flows, error announcement alone can be invoked, thus allowing the provider to monitor sustained QoS over the communication channel with respect to error rates.

In our work, we define a reliability enumeration type for specifying the type of mechanism required from the provider in dealing with the continuous media flow or sub-flow. Users may specify to have no error control mechanism instantiated; to have lost or corrupted PDUs replaced with zeros or replaced with the previous correctly received PDU; to have errors announced; and finally to have errors in transmission corrected. For correcting errors in transmission, forward error recovery schemes are employed. Retransmission schemes are not suitable for continuous media data as they increase experienced end-to-end delay and disrupt continuity in media playout. Because forward error recovery schemes do not require feedback from the client to the source, each client in a point-to-multipoint session can select the 
instantiation of recovery mechanisms independently from other clients (if so desired and albeit to multipeer integrity constraints).

Multipeer integrity specifies conditions on the number and/ or identity of participating entities. Connection establishment conditions state the number and/ or identity of recipients who have to respond positively to the connect request. In the data transmission phase integrity conditions refer to the number and/ or identity of recipients who have to receive a "correct copy" of the transmitted data*. For continuous media data, these constraints are tied to all QoS parameters and do not just apply on a single data packet. In other words, as long as the QoS specified for the recipients in the identified sub-set is met data transfer is deemed successful. In our model quorum, $k$ (specified number of receivers) and mandatory (list of mandatory receivers) are the integrity conditions that can be applied to both connection establishment and data transfer. Integrity conditions for the data transfer phase can be dynamically changed in a orderly manner. For instance, if a receiver who was required for one half of the communication wishes to leave. In this case the integrity conditions have to be changed rather than to release and establish the communication again.

Table 1 QoS dependencies

\begin{tabular}{|c|c|}
\hline Metric & Behaviour \\
\hline Throughput & $\begin{array}{l}\text { Network path/ arm dependent. If filters such as frame droppers, codec or } \\
\text { mixers are inserted somewhere in the path the throughput delivered to } \\
\text { individual clients may not be the same. In sub-flow selection, the } \\
\text { throughput is the same for each client consuming the particular sub-flow. } \\
\text { Note: sub-flows can be further filtered (if required) and then once again } \\
\text { throughput delivered to clients may be different. }\end{array}$ \\
\hline Delay & $\begin{array}{l}\text { Each client can experience different delays depending on path and } \\
\text { network arm characteristics. Also, some filtering operations may incur } \\
\text { further processing overheads. Some applications may require that this } \\
\text { delay is bounded across multiple clients. }\end{array}$ \\
\hline Jitter & $\begin{array}{l}\text { Dependent on the burstiness induced on traversing network paths/ arms. } \\
\text { Experienced jitter is thus different for each client and smoothing } \\
\text { capabilities depend on available buffer resources. Again, this may need to } \\
\text { be bounded across multiple clients. }\end{array}$ \\
\hline Error Rate & $\begin{array}{l}\text { Experienced error rates are different for each client, they depend on the } \\
\text { properties of the network path/ arm traversed. }\end{array}$ \\
\hline Reliability & $\begin{array}{l}\text { It is up to individual clients to detect and recover from transmission } \\
\text { errors. FEC techniques are used with continuous media flows. }\end{array}$ \\
\hline Agreement & $\begin{array}{l}\text { Agreement levels reached is dependent on network path/ arm traversed, } \\
\text { thus this is independent for different clients. Some applications may } \\
\text { however enforce that a session is not open unless an identified subset of } \\
\text { clients are capable of getting the same service commitment. }\end{array}$ \\
\hline
\end{tabular}

During the establishment of a point-to-multipoint connection, the QoS characteristics of a flow may be modified through a path or on a particular network arm to reflect its capabilities or client requirements. In Table 1 we highlight the possible behaviour of each metric in a point-tomultipoint service. Threshold metrics are only used at client end-systems for the implementation of adaptive QoS mechanisms. However, depending on the multipeer integrity associated with data transfer, the delivered QoS to other clients may be influenced through the actions taken by a single client.

\footnotetext{
* Many authors refer to this as reliability, multicast reliability or degree of reliability (Mathy 1994), (Schutt 1993). We deliberately chose the term 'multipeer integrity' to distinguish it from reliability as it is defined above.
} 


\subsection{Filter operations}

The concept of filtering continuous media data is becoming an accepted form of meeting distinct QoS capabilities and requirements for multipeer data flows. Filtering mechanisms at Lancaster are applied to codec generated data. These mechanisms exploit and adapt the structural composition of this type of continuous media traffic to ensure that end-user, application, endsystem, network capabilities and requirements are met. Distinct approaches to filtering can be taken according to the particular problem being addressed. These include: optimisation of bandwidth usage, adoption of filters for handling client heterogeneity, optimisation of resource allocation, optimisations in the retrieval process of stored media, etc. Filter operations are always performed at the edge of the network (e.g. above IP or AAL5). These operations are instantiated at the most optimal point in the multicast tree.

\section{FLOW MANAGEMENT MODEL}

Flow management is concerned with the establishment, maintenance and release of continuous media data flows along the various network paths for both point-to-point and point-tomultipoint services. The general model assumes a client/ server paradigm, objects or devices which produce continuous media data are classified as sources, while those that consume it are classified as clients. In the following sections we describe the three main modules making up our flow management model which include flow specification, flow establishment and flow maintenance. Flow release is discussed as a side issue relating to both flow establishment and maintenance.

\subsection{Flow specification}

Flow characterisation including quantitative QoS, agreement levels, actions on thresholds, reliability requirements, and appropriate filter operation identification are specified by the application and are contained within a flow specification (FlowSpec) structure. This structure can identify individual sub-flow requirements for hierarchical encoding. The communications sub-system will treat the sub-flows independently in terms of throughput, error rates, reliability requirements and delays.

In a multi-client environment the FlowSpec characterises how the data flow originates from the data source. Through filtering mechanisms the QoS of the flow can change on its way to the data client(s). Moreover, the flow received by each client might have different QoS characteristics. It is therefore feasible for clients to provide a client specific flow specification (client-FlowSpec) describing their particular QoS requirements and characteristics. Thus, depending on application policies, and mechanisms and functions provided by the service provider, the FlowSpec and the client-FlowSpec(s) can be different (or not).

\subsection{Flow establishment}

For flow establishment we have adopted a simple scheme which minimises QoS negotiation and hence simplifies flow establishment. Essentially we work with pre-defined profiles for continuous media data flows which are suitable for particular applications. These are depicted by appropriate FlowSpecs. These FlowSpecs contain discrete quality values as in (Wolf, 1994), rather than using more complex continuous ranges as described in (Mathy, 1994). Through the instantiation of filters, these profiles can be split so that they support a finite set of discrete quality playout levels. These discrete levels can be represented through the aide of FlowSpecs which typically characterise client requirements (i.e. client-FlowSpec(s)). Three of the low level modules involved in flow establishment are illustrated in Figure 1. 




Figure 1 Flow Establishment Model.

The resource management module is made up of three components: admission control, filter allocator and packet scheduler. The functionality supported by each of these components depends on where they reside and execute. For example, intermediate nodes like routers will make resource reservation and allocation decisions based on simple policies. On the other hand, end-systems may make decisions based on much more complex policies as they need to handle a much broader view which encapsulates the application requirements, computing platform support (including devices, operating system, interconnect architecture, etc.), communication protocol support, network interfaces, etc.

Typically a client joins a point-to-multipoint service by registering with some group communication management entity (Mauthe, 1994). This group management entity invokes flow establishment by contacting the appropriate resource management module at a particular source end-system. Flow establishment then takes place between the source and the client through the aide of a handshake scheme which employs a resource reservation and allocation protocol akin to RSVP (Zhang, 1993), and ST-II (Topolic, 1990).

At each node in the forward path, admission control tries to reserve resources based on the source's FlowSpec. Admission control will always attempt to reserve resources for the highest quality first. If it can not, it will attempt to allocate some filter which will reduce the resource requirements to the next highest quality level. The QoS characteristics in the forwarded FlowSpec are of course adjusted to reflect this new level of QoS. This proceeds until the FlowSpec reaches the client. The client can then decide whether to accept the level of QoS presented to it or whether it wishes to reduce it to some other level (through appropriate filtering) to reflect its capabilities. If a simple acceptance is made, on the backward path admission control (at each intermediate node) allocates the reserved resources and invokes the packet scheduler accordingly. If a lower level QoS is required (reflected in the client'sFlowSpec), on the backward path appropriate resources are again allocated making sure that over committed resources are relinquished. A point is found in the dissemination tree at which the appropriate filter can be invoked to provide the requested QoS level without affecting the $\mathrm{QoS}$ of other parties involved in the point-to-multipoint service. The client-FlowSpec is still however relayed back to the source.

The source decides based on the incoming replies from the clients and the multipeer integrity conditions if the connection is successfully established or not. The establishment is deemed to be successful when the necessary sub-set of clients specified in the integrity conditions can get the required QoS. The source sends a confirmation to all those clients which can participate in the session and a release to those whose QoS requirements can not be fulfilled. Provisionally allocated resources for the latter are released. In an unsuccessful attempt to establish a connection, i.e. the multipeer integrity conditions are not fulfilled, the source sends a disconnect to all clients. All provisionally allocated resources are released.

Flows made up of a subset of subflows (e.g. hierarchically encoded video) can also be set up in the same way. Each subflow is treated independently for resource reservation and allocation where the lowest level subflow provides the minimal quality media playout level and 
the others enhance this level to a better quality. Subflows are uniquely identified by a subflow_id which also indicates its position in the hierarchy. During the flow establishment phase, a resource management module (as described) will attempt to first meet the requirements of all subflows. If it can not, it will try to meet that of the next quality level by dropping the highest subflow_id and so on.

\subsection{Flow management}

To manage flows, like in flow establishment, a generic resource management module is employed as illustrated in Figure 2. In this model we have included a new component called a monitor. The premise we work on is that QoS is monitored at the end-systems, in other words at the origin (source) and destination (client) of the continuous media data flow. Typically it is the clients whom are interested in the delivered QoS and hence the clients perform all monitoring except in particular interactive applications where feedback is employed to adjust flows at the origin. Of course, some networks services will perform their own monitoring and flow management but our interests are on end-to-end QoS and we build on top of the quality provided by the underlying network service to raise this quality (if possible) to a level acceptable to the user.

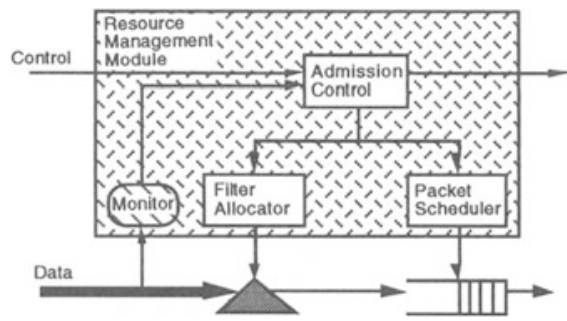

Figure 2 Flow management model.

In general, the flow management module monitors QoS metrics over default or user supplied sample periods. If associated qualitative agreements are breached, monitors inform admission control. Depending on the policies associated with the flow admission control may attempt to adapt the flow characteristics through the insertion of filters at an appropriate link in the dissemination tree and adjust packet scheduling in accordance to these changes. Such changes can only be instantiated if they do not alter multipeer integrity related issues or the QoS supplied to other clients consuming the same flow.

Four different levels of agreement which can be reached between transport provider and client with respect to particular QoS are considered. The best effort semantic, implies that the user is not concerned with the level of QoS delivered for a particular metric and/ or that the provider can not give anything but a best effort service because of the underlying network support or end-system resource availability. Best effort with threshold is practically the same as above but the user has identified a threshold which the provider will monitor and if breached the associated action will be invoked. The main action of interest here is that of adapt. For example filters can be instantiated which will reduce the playout quality to another level, possibly alleviating the congestion problem while providing a playout quality which is still acceptable to the user (Yeadon, 1994).

The compulsory semantic is used to control the number of levels to which a specific FlowSpec profile can be reduced and yet maintain an intelligible playout quality. Beyond that level, the video or audio data is non-intelligible and the user does not wish to maintain the connection open. For the compulsory semantic a threshold can also be defined which gives the provider a chance to improve the quality before actually releasing the connection. Finally, metrics with guaranteed semantics can only be satisfied through appropriate resource allocation 
along the network path all the way up to the particular client and at the end-system. Threshold metrics may also be specified along side guaranteed metrics but these are only employed for signalling purposes to allow higher level application functionality such as flow synchronisation (possibly across multiple clients) to be maintained.

\section{THE PROTOCOL SUITE}

The flow management model presented above is being evaluated through the development of an appropriate protocol suite. This protocol suite is illustrated in Figure 3. Essentially we have taken the supplied TCP/ IP protocol suite and inserted a Rate-Based Packet Scheduler (RBPS) between the IP protocol and the network driver to shape traffic. Traffic shaping is used to smooth VBR sources towards a more constant data bit stream which allows better utilisation of resources (Kim, 1995).

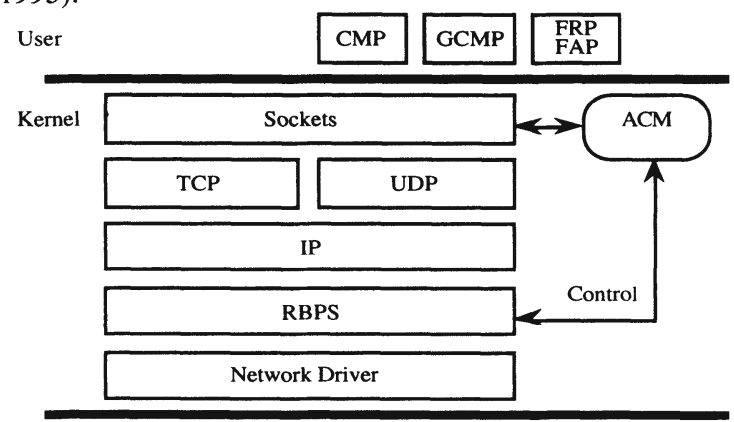

Figure 3 Protocol stack

We have also included an Admission Control Module (ACM) which at present only performs a buffer, network bandwidth and delay test on all new continuous media connection requests. The UDP protocol is utilised by the Continuous Media Protocol (CMP) as well as by the Group Communication Management Protocol (GCMP) and a combined Flow Reservation and Filter Allocation Protocols (FRP/ FAP). CMP, GCMP and FRP/ FAP are represented as user level libraries. IP multicast addresses are obtained prior to multipeer communications establishment by the GCMP. For control messages which need to be interpreted by intermediate nodes the protocol field in the IP header is used to identify both FRP and FAP messages. We are currently up-dating the IP protocol to IPv6 (IPv6-IPng, 1994) which provides flow identification facilities for which flow specs are held by routers to determine which resources should be made available for each flow. This simplifies our FAP and FRP. The task of GCMP is twofold, it is responsible for dynamic join and leave of new candidates in multipeer data flow and, in our current implementation, it also validates integrity conditions during an on-going communication. GCMP co-ordinates these processes which involve CMP and FRP/ FAP.

The CMP protocol provides two interfaces to user processes utilising two separate sockets, one for control data and the other for continuous media data. This is in line with previous work on transport protocols at Lancaster (Garcia, 1993), (Coulson, 1991) and is akin to the current RTP proposal (Schulzrinne, 1993). The control interface is employed to signal results of QoS monitoring and invoke actions (via feedback) at the source or intermediate nodes, in the dissemination path of the continuous media stream, which alter its characteristics to reflect network and/ or end-system capabilities. GCMP, FRP and FAP messages are also transmitted through the control path.

This separation allows for simple migration towards a better performance architecture which will incorporate hardware supported decoding and playback facilities. As described in (Fall, 1994), in-kernel data paths are exploited to minimise both data copies and context 
switches. This involves intercepting protocol upcalls when data is delivered from the network and redirecting this data to either an in-kernel buffer or directly to the decoder's frame buffer (if the hardware used supports DMA transfers). With the in-kernel buffer scheme, a buffer is shared between the network driver and the decoder driver allowing these devices to function asynchronously without user process interaction to control the data transfer across these devices through explicit read and write system calls. Under this set-up, continuous media data does not cross the user/ kernel boundary and only the control socket interface is utilised by user processes. This is suitable for multimedia applications which simply require decoding and playback functionality but have no requirements for performing any further processing on the delivered continuous media data.

\section{EXPERIMENTAL RESULTS}

Filtering provides the means to accommodate distinct QoS characteristics to support multipeer communications within heterogeneous environments. Thus, it is essential to know the quantitative effects on a bit-stream of instantiating a filter operation before it can be effectively used. This is primarily because the results determine when and why a particular filter may be used, but also if there are any adverse side effects of providing a particular filtering function. Preliminary research has concentrated on: the effects on the size of the compressed bit-stream being operated upon, the processing requirements (in terms of time) of the filter operations themselves, subsequent effects on decoder requirements and to a lesser extent the subjective differences observed on the decompressed information.

\subsection{Bit rate reductions}

Our current research has produced some interesting results concerning the bit-rate reductions achieved with our present filter mechanisms, namely the frame based dropping filter, the colour to monochrome converter and the coefficient based low-pass frequency filter. Also, an unexpected effect of the transcoding filter was observed.

The work with the frame dropping filter has proved the importance for a good flow spec when establishing an adaptive communication path. Because of the varied structure of an MPEG (MPEG-1, 1993), (MPEG-2, 1994) video stream, in that, no set patterns or ratios of I-, P-, and B-pictures are specified within the standard, the effects of dropping frames can produce quite diverse results. We have begun to tie down the relationship between stream structure and bit-stream size by performing dropping operations on, and analysing, a number of MPEG sequences. These sequences were produced by our own equipment and gathered from various MPEG archive sites around the Internet. Figure 4 shows the relative amounts of the bit-stream taken up by I-, P-, and B-pictures in a number of these test files, and how the group of pictures structure affects these ratios.

Although a stream may contain many more B-pictures than I-pictures, because of the relative sizes of the pictures, the actual amount of data used to represent one I-picture may be the same as to represent a number of B-pictures (P-picture sizes lie between the two). This is important to consider when performing frame dropping operations as there is a non-linear relationship between the frame rate and bit-rate that is dependent on this GOP structure. For example, in Figure 4 (a) dropping P- and B-pictures reduces the bit-rate by around $60-70 \%$ and frame rate by $5 / 6$ ths whereas in Figure 4 (b) the same operation reduces the bit-rate by a comparable 70 $80 \%$ but reduces the frame rate by $14 / 15$ ths. A finer control of bit-rate is achieved if only some of the B-pictures or P-pictures are dropped; Figure 5 (a) shows the possible steps of frame rate reduction and how much the bit-rate drops per frame, in terms of percentage of the total bitstream. 


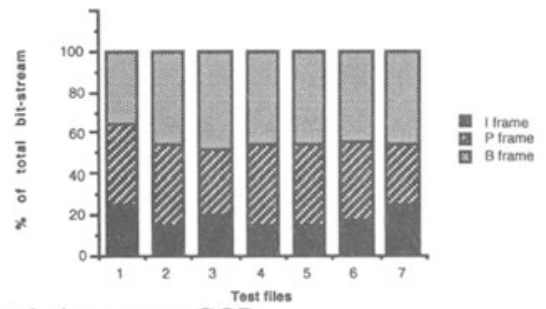

(a): 6 pictures per GOP

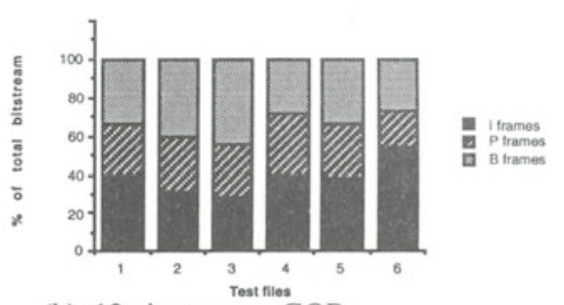

(b): 12 pictures per GOP

Figure 4 Correlation of bit-stream to picture type

The values shown are averages taken from the test-files shown in Figure 5 (a). Two kinks exist in each line where the frame type changes from B- to P-picture and from P-picture to Ipicture. This is where the step sizes change due to the size of the frames being dropped changing (i.e. B-pictures are smallest and most numerous so provide finer alterations to the bitstream).

The effect of colour-monochrome filtering, removing the colour information, is easy to predict when dealing with uncompressed video streams (i.e. RGB data is summed together giving a third of the data size and YUV has the $U$ and V components removed so, depending on the component ratio either a third $(4: 2: 0)$, a half $(4: 2: 2)$ or two thirds $(4: 4: 4)$ of the data is removed). MPEG 1 video employs a 4:2:0 component ratio between the luminance and colour components, so it is expected that removing the colour components would reduce the bit-stream by a third. In our experiments we found that the compressed colour information only occupied a sixth of the compressed MPEG 1 video bit-stream when passed though the colour to monochrome filter.



(a) Dropped frames to bit-stream ratio Figure 5 Filter Effects

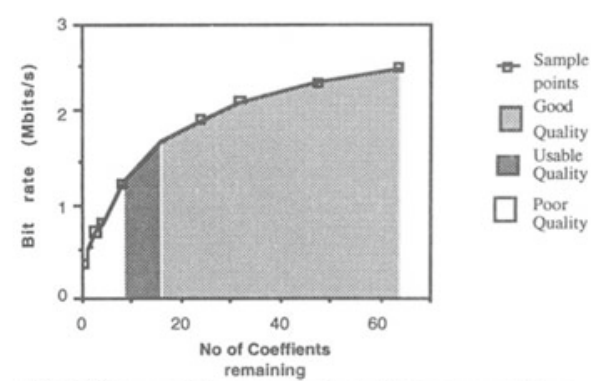

(b) Effect on bit-stream size of low-pass filter

Currently in operation is the Motion-JPEG to MPEG 1 video transcoder. This operates by using the JPEG entropy decoding scheme (JPEG, 1993) to reach a semi-decompressed state and then recompressing the bit-stream using the MPEG entropy encoding scheme; producing an I-picture only MPEG 1 video stream. When transcoding the set of Motion-JPEG test files we noticed a slight increase in the bit-stream size of about $2 \%$. This is probably because the JPEG entropy encoding tables can be tailored to provide the best compression ratio for a particular set of input data, whereas MPEG 1 video employs a generic entropy table that achieves good results for practically all cases. Although the difference is not large it is important to be aware of any such possible changes when allocating resources. 
The low-pass filter operates by discarding higher frequency coefficients from each block in each frame. The effect of the filter depends on how much high frequency component exists within an image. The FDCT and quantization procedures are intended to leave much of the block and in particular the higher frequency components as zero. Therefore, as most of the information about a picture exists in the low frequency areas the impact of the filter is greatest in this region. Figure 5(b) shows the effect of the low-pass filter on a Motion-JPEG sequence.

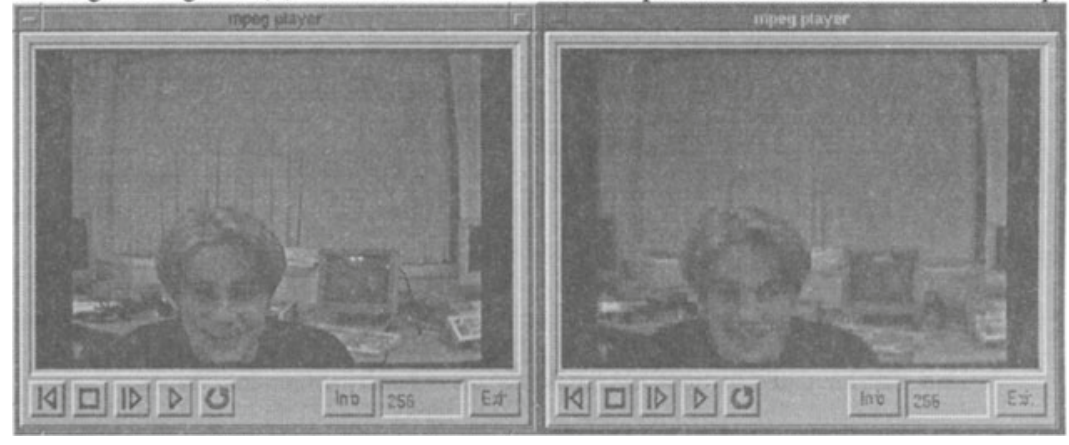

(a): Full quality

(b): 4th coefficient cut-off

Figure 6 Picture quality after applying low pass filter

The quality of the decompressed picture inevitably decreases as more information is discarded; the image becomes less sharp as the high-frequency components are removed. In this case the image quality remained quite good down to about 16 coefficients and still quite usable down to around 8 coefficients. The image quality is clearly reduced with a 4 th coefficient cutoff but might still be acceptable for many application as can be seen in Figure 6.

\subsection{Real-time requirements}

Just as it is important to know the effects of a filter on the bit-stream size, and hence throughput requirements, the effects on delay and jitter are also essential. The processing requirement of each operation also allows us to evaluate how scalable the prototype system is. The following section details the speed of current filter operations and indicates the impact of these filters on stored and live video delay requirements. The MPEG 1 video player referred to below is based on the Berkeley MPEG player (Rowe, 1994). The times given are for total processing time.

The transcoder and low-pass filter are the most computationally intensive operations of the present filter functions and hence require a greater amount of processing time. Both filters must perform entropy decoding before executing the filter algorithm and entropy encoding following the operation. It is this entropy decompression and recompression that accounts for the main proportion of the processing time.

The transcoder can convert a relatively large Motion-JPEG file, ( 252 frames $320 \times 240$ pels) to an I-picture only MPEG sequence at a rate of about 5 frames/sec. This may seem quite limiting but the software MPEG player we are using plays this same sequence at only 3-4 frames/sec (on our 486 machines), so this is of a comparable speed.

The low-pass filter discards information, the more it discards the less it has to process for reencoding and hence the faster it operates. Again due the entropy stage of the filter operation it is of similar speed to the transcoder, but if the filter cut-off is 8 coefficients (i.e. all but the first 8 coefficients are removed) the filter runs at around 8 frames/sec. Currently the low-pass filter is only designed for Motion-JPEG and I-picture only MPEG video but the next stage is to improve the filter to operate on the full set of MPEG 1 video picture types which will improve the frame rates achieved. 
The frame level filters involve a relatively simple processing scheme which mainly require scanning for header codes and structuring the data accordingly. The parsers' speed is dependent on how much detail and information the operation is trying to extract. Scanning an MPEG 1 video file can currently be done at 170-190 frames/sec depending on frame size. If an MPEG sequence were to be stored this scanning and subsequent flow-spec generation would be performed at write time and the flow-spec stored with the sequence. To scan a 1758 frame $144 \times 112$ pel MPEG 1 video takes about 10 seconds which at time of storage is negligible but more significant if performed at playback.

The mixer and dropper operate at a slightly slower rate than the parser due to the increase in data that has to be moving around. The mixer is currently designed for I-picture only MPEG 1 video streams of the same size, so the decoder does not have to be reinitialised for each frame hence slowing down playback. In fact the software MPEG player used ignores any attempts, in the form of sequence headers, to change the decoding parameters during playback and often will suffer unrecoverable failure.

The dropper operates faster when actually performing more filtering because once a frame has been dropped there is no longer any processing required on that frame. In tests removing all B-pictures (142 frames) from a 215 frame $320 \times 180$ pel MPEG sequence was processed at a rate of 185 frames/sec and removing both B- and P-pictures (54 frames) could be done at 194 frames/sec. The source filter performs dropping on an assembled MPEG 1 video sequence, in that it must first scan for frame headers to delimit each frame. The network dropper needs only to look in the packet header for the frame type and hence performs at a much faster rate.

We have deduced that filtering operations are possible in a real-time environment, if at a slight cost in end-to-end delay. The saving on network throughput may in fact counteract the increase in network delay experienced. We have shown some of the results of our filter operations in the form of stills but a set of MPEG video sequences demonstrating the effects of filtering, and the experimental software used to perform the filtering operations described here, can be found at http//:www.comp.lancs.ac.uk/computing/users/njy/demo.html.

\section{CONCLUSIONS}

Distributed multimedia applications have diverse characteristics and requirements arising from the nature of multimedia traffic. In a true open environment discrete and continuous media data has to be freely exchanged between a group of users located at distinct geographical locations. Each user's capabilities to process such data is determined by the capability of his/ her computing machine and the characteristics of the underlying network. The inherent properties of such applications require support in both group communications and QoS.

QoS support for audio and video multipeer communication is distinguished from common QoS provision through the number of participants and their distinctive, individual QoS requirements. To overcome this problem we employ filters to provide individual QoS for individual clients. These filters operate on encoded data flows which are characterised by distinct, discrete quality values. Therefore we work with a set of pre-defined profiles for continuous media data flows. Through the instantiation of certain filters, these profiles can be split so that they support a finite set of discrete quality playout levels. To validate the success of data transmission, certain integrity conditions have to be fulfilled. These conditions give the identity and/ or number of receivers that have to receive the data flow according to their own QoS specifications.

We found that none of the current technologies or standards meets the requirements of distributed multimedia applications. They usually lack the necessary QoS and/ or group support. In this paper we introduce a flow management model based on the previous model developed for peer-to-peer multimedia communication at Lancaster and show how it can be supported at different layers of the system architecture. The data flow is established from the source to the clients. During the establishment phase all the necessary reservations and instantiations of filters are made. The flow is maintained according to the client specifications. 
Any action to maintain QoS has to consider multipeer integrity conditions. Our model is being evaluated through appropriate developments of the protocol suite that we have reported. We have also given initial experimental results obtained from the use of our filter operations.

Our experience with the use of filters has shown that it is possible, in a heterogeneous environment, to meet the distinct requirements of different participants within distributed multimedia applications. QoS can be adapted to a certain degree in this cases where networks and receivers have different capabilities and distinct requirements. In our model the concept of multipeer integrity is used to establish success or failure of the communication in an open environment based on individual requirements and capabilities. We are currently working on an extension of our model to allow multimedia conferencing between multiple senders and receivers.

\section{ACKNOWLEDGEMENTS}

The work reported in this paper is carried out in the context of the QoS-A project funded by the UK Engineering and Physical Sciences Research Council (grant number GR/H77194) and in collaboration with GDC Advanced Research (formerly Netcomm Ltd). This work is also carried out in the context of the GCommS project funded by the EPSRC (grant number GR/J47804).

\section{REFERENCES}

Campbell, A., Coulson, G., and Hutchison D. (1994) A Quality of Service Architecture. ACM Computer Communications Review.

Coulson, G., Garcia, F., Hutchison, D. and Shepherd D. (1991) Protocol Support for Distributed Multimedia Applications. Proceedings of 2nd International Workshop on Network and Operating Systems Support for Digital Audio and Video, Heidelberg, Germany.

Coulson, G., Blair, G.S., Stefani, J.B., Horn, F. and Hazard, L. (1995) Supporting the RealTime Requirements of Continuous Media in Open Distributed Processing. Computer Networks and ISDN Processing, Vol. 27.

Danthine, A., Baguette, Y., Leduc, G. and Leonard, L. (1992) The OSI 95 Connection-mode Transport Service - The Enhanced QoS. Proc. 4th IFIP Conference on High Performance Networking, Liege, Belgium.

Fall, K. and Pasquale, J. (1994) Improving Continuous-Media Playback Performance with InKernel Data Paths. Proceedings of International Conference on Multimedia Computing and Systems.

Garcia, F. (1993) A Continuous Media Transport and Orchestration Service. Ph.D. Thesis, Department of Computing, Lancaster University, Lancaster LA1 4YR, UK.

Henckel, L. (1994) Multipeer Transport Services for Multimedia Applications. Proc. 5th IFIP Conference on High Performance Networking, Grenoble.

IPv6-IPng (1994) Hinden, R., Internet Protocol, Version 6 (IPV6) Specification. InternetDraft, draft-hinden-ipng-ipv6-spec-00.txt.

JPEG (1993) ISO IEC JTC 1/SC29 Information Technology - Digital Compression and Coding of Continuous-Tone still Images. International Standard ISO/IEC IS 10918.

Kim, K., Garcia, F. and Hutchison, D. (1995) A Rate Control Mechanism for Continuous Media Traffic. Proc. of 2nd Communication Networks Symposium, Manchester.

Mathy, L. and Bonaventure, O. (1994) QoS Negotiation for Multicast Communications. Proc. International COST 237 Workshop on Multimedia Transport and Teleservices, Vienna, Austria.

Mauthe, A., Hutchison, D., Coulson, G. and Namuye, S. (1994) From Requirements to Services: Group Communication Support for Distributed Multimedia Systems. Proc. 2nd 
International Workshop on Advanced Teleservices and High-Speed Communication Architectures (IWACA'94), Heidelberg, Germany.

MPEG-1 (1993) ISO IEC JTC 1/SC29, Information Technology - Coding of Moving Pictures and Associated Audio for Digital Storage Media up to about 1.5Mbit/s. International Standard ISO/IEC IS 11172.

MPEG-2 (1994), ISO IEC JTC 1/SC29, Information Technology - Generic Coding of Moving Pictures and Associated Audio Information. Draft International Standard ISO/IEC DIS 13818.

Partridge, C. (1993) Gigabit Networking. Addison-Wesley Professional Computing Series, ISBN 0-201-56333-9.

Pasquale, G., Polyzos, E., Anderson, E. and Kompella, V. (1992) The Multimedia Multicast Channel. Proc. 3rd International Workshop on Network and Operating System Support for Digital Audio and Video, San Diego, USA.

Robin, P., et al. (1994) Implementing a QoS Controlled ATM based Communication System in Chorus. Proc. 4th IFIP International Workshop on Protocols for High Speed Networks.

Rowe, L., Patel, K., Smith, B., and Liu, K. (1994) MPEG Video in Software: Representation, Transmission, and Playback. Proc. High-Speed Networking and Multimedia Computing, San Jose, California.

SC21/1 (1995) Basic QoS Framework. ISO IEC JTC 1/SC21, Available by anonymous ftp from ftp.comp.lancs.ac.uk in directory pub/ISO_QOSF.

Schulzrinne, H. and Casner, S. (1993) RTP: A Transport Protocol for Real-Time Applications. Work in Progress, Internet Draft, <draft-ietf-avt-rtp-04.ps>.

Schuett, T., Sandvoss, J. and Steinmetz, R. (1993) XTP and Multimedia. in IEEE Global Telecommunications Conference, Communication for a Changing World, Houston, Texas.

Stefani, J.B. (1993) Computational Aspects of QoS in an Object Based Distributed Architecture. 3rd Int. Workshop on Responsive Computer Systems, Licoln, New Hampshire.

Topolic, C. (1990) Experimental Internet STream Protocol, Version 2 (ST-2). Internet, RFC 1190.

Wolf, L.C., Herrtwich, R.-G. and Delgrossi, L. (1994) Filtering Multimedia Data in Reservation-Based Internetworks. Technical Report 43.9408, IBM European Networking Centre, Heidelberg.

Yeadon, N., Garcia, F., Campbell, A. and Hutchison, D. (1994) QoS Adaptation and Flow Filtering in ATM Networks. Proc. 2nd International Workshop on Advanced Teleservices and High-Speed Communication Architectures (IWACA'94), Heidelberg, Germany.

Zhang, L., et al. (1993) A New Resource ReSerVation Protocol. Work in Progress, Internet Draft, <rspv.ps.Z>. 